\title{
Unerwünschte Arzneimittelwirkungen
}

\section{Pharmacogenetics}

Sowohl einzelne Patienten als auch bestimmte Bevölkerungsgruppen reagieren auf bestimmte Arzneimittel abweichend vom Großteil der Bevölkerung. Solche unerwarteten Arzneimittelwirkungen aufgrund eines veränderten Arzneistoffmetabolismus sind häufig genetisch bedingt. Es können sowohl verstärkte als auch abgeschwächte Wirkungen resultieren. Diesen Wirkungsdifferenzen liegen häufig - monogen vererbte - genetische Polymorphismen zugrunde $(1,2,16,17)$. Diese sind vor allem dann bedeutsam, wenn die betreffenden Arzneimittel eine geringe therapeutische Breite haben und/oder es zu Wechselwirkungen an dem betroffenen Enzym kommt. Des Weiteren ist die Stellung des Enzyms im Gesamtmetabolismus und die eventuelle Bildung aktiver Metabolite von dem betreffenden Arzneistoff von Interesse. Die therapeutischen Konsequenzen müssen daher für jedes Pharmakon isoliert betrachtet werden.

kurzgefasst: Die Bedeutung eines genetischen Polymorphismus hängt ab von der therapeutischen Breite des Arzneimittels, von der Stellung des Enzyms im Gesamtmetabolismus und von der möglichen Bildung aktiver Metaboliten des betreffenden Arzneimittels.

Polymorphismen im Fremdstoffmetabolismus sind bis zum Zeitpunkt der Belastung des Organismus durch Arzneimittel oder andere Fremdstoffe klinisch inapparent, allerdings sind Zusammenhänge zwischen polymorph exprimierten Enzymen und malignen oder degenerativen Erkrankungen beschrieben werden $(5,7,12,14)$.

\section{Historischer Albriss}

Schon Pythagoras erkannte die Gefahr des Genusses von FavaBohnen für einige Menschen. 1956 wurde der Zusammenhang zwischen Erleiden eines hämolytischen Ikterus nach Behandlung mit den Antimalariamitteln Primaquin und Chloroquin auf einen Mangel des Enzyms Glucose-6-Phosphat-Dehydrogenase (G6PD) zurückgeführt, welcher auch für die hämolytischen Krisen nach Fava-Bohnen verantwortlich ist. Dieses X-chromosomal vererbte Enzym spielt ebenfalls in der Behandlung mit vielen Pharmaka, wie z.B. mit Sulfonamiden, Dapson, Nitrofurantoin, Chinidin, Thiopental, Probenecid und Acetylsalicylsäure, eine Rolle. In der mitteleuropäischen Bevölkerung weisen weniger als 0,05\% einen Mangel an G6PD auf, in einigen Mittelmeerländern bis zu 50\% $(17,22)$.

\section{kurzgefasst: Die Art und Häufigkeit genetischer Polymor-} phismen variiert zwischen ethnischen Gruppen.

Schnell wurde bei dem 1951 eingeführten Succinylcholin erkannt, dass bei einigen Patienten protrahierte Atemlähmungen (Succinylcholin-Apnoe) auftraten. Kalow und Genest beschrieben bereits 1957 einen Zusammenhang mit dem Vorkommen einer atypischen Variante des Enzyms Pseudocholinesterase, die Succinylcholin nicht in der üblichen Geschwindigkeit hydrolysiert (17).

1953-1960 wurden sowohl von deutschen als auch amerikanischen Forschergruppen große interindividuelle Unterschiede in der Pharmakokinetik von Isoniazid beschrieben. Verantwortlich hierfür ist das Enzym N-Acetyltransferase (NAT2). Etwa die Hälfte der europäischen Bevölkerung und 70-90\% der Japaner, Chinesen und Eskimos inaktivieren Isoniazid rasch durch AcetyLehrstuhl für Klinische Pharmakologie der Universität Witten/Herdecke 
Tab.1 Genetische Polymorphismen und deren klinische Bedeutung außerhalb des Cytochrom-Systems; mod. n. (19).

\begin{tabular}{|c|c|c|}
\hline Enzym & Substrate & klinische Bedeutung \\
\hline \multicolumn{3}{|l|}{ Alkohol-DHG } \\
\hline Aldehyd-DHG & Ethanol & Alkoholunverträglichkeit \\
\hline Aldehyd-DHG & $\begin{array}{l}\text { Cyclophosphamid, } \\
\text { Ifosfamid }\end{array}$ & Nebenwirkungsprofil \\
\hline NAT-2 & $\begin{array}{l}\text { Dapson, Isoniazid } \\
\text { Procainamid, Amrinon } \\
\text { Sufamethoxazol } \\
\text { Hydralazin }\end{array}$ & $\begin{array}{l}\text { große Variabilität bei } \\
\text { Wirkung, Nebenwirkun- } \\
\text { gen und Interaktionen }\end{array}$ \\
\hline NAT-2 & & $\begin{array}{l}\text { Arzneimittel-induzierter } \\
\text { Lupus; Schwankung } \\
\text { der antihypertensiven } \\
\text { Wirkung }\end{array}$ \\
\hline $\begin{array}{l}\text { Dihydropyrimidin- } \\
\text { DHG }\end{array}$ & $\begin{array}{l}\text { 5-Fluor-Uracil, } \\
\text { einige Virustatika }\end{array}$ & verstärkte Toxizität \\
\hline G6PD & $\begin{array}{l}\text { Sulfonamide, } \\
\text { Primaquin, Chloroquin } \\
\text { Dapson, Nitrofurantoin, } \\
\text { Chinidin, Thiopental, } \\
\text { Probenecid, ASS, u.v.m. }\end{array}$ & Hämolyse \\
\hline$M E$ & $\begin{array}{l}\text { Carbamazepin, } \\
\text { Phenytoin }\end{array}$ & Nebenwirkungsprofil \\
\hline $\begin{array}{l}\text { Met-Hb- } \\
\text { Reduktase }\end{array}$ & $\begin{array}{l}\text { Anilin, Chloramphenicol, } \\
\text { Nitrate, Sulfonamide, } \\
\text { u.v.m. }\end{array}$ & Methämoglobinämie \\
\hline PCE & Succinylcholin & $\begin{array}{l}\text { prolongierte } \\
\text { Atemlähmung }\end{array}$ \\
\hline TPMT & $\begin{array}{l}\text { Azathioprin, } \\
\text { 6-Mercaptopurin }\end{array}$ & erhöhte Toxizität \\
\hline Thiol-S-MT & $\begin{array}{l}\text { D-Penicillamin, } \\
\text { Captopril }\end{array}$ & $\begin{array}{l}\text { große Variabilität bei } \\
\text { Nebenwirkungen, Wir- } \\
\text { kung und Interaktionen }\end{array}$ \\
\hline Catechol-O-MT & $\begin{array}{l}\text { L-Dopa, Alpha- } \\
\text { Methyldopa }\end{array}$ & Variabilität bei Wirkung \\
\hline GST & $\begin{array}{l}\text { Umweltgifte, halogen- } \\
\text { haltige Lösungsmittel } \\
\text { NO-liberierende } \\
\text { Pharmaka }\end{array}$ & erhöhte Toxizität \\
\hline \multicolumn{3}{|c|}{$\begin{array}{l}\text { DHG = Dehydrogenase; NAT-2 = (Arylamin-)N-Acetyltransferase 2; } \\
G 6 P D=\text { Glucose-6-Phosphat-Dehydrogenase; } \\
\text { ME = Mikrosomale Epoxihydrolase; } P C E=\text { Pseudocholinesterase; } \\
T P M T=\text { Thiopurin-S-Methyltransferase; GST = Glutathiontransferas }\end{array}$} \\
\hline
\end{tabular}

lierung, der Rest der Bevölkerung deutlich langsamer (schnelle und langsame Acetylierer). Etwa 90\% der Patienten mit Sulfonamid-induzierten Nebenwirkungen (z.B. nach Gabe von Cotrimoxazol) sind langsame Acetylierer (18).

In den 70er-Jahren wurden von zwei Forschergruppen (Eichelbaum et al; Mahgoub et al) erste Hinweise auf verstärkte unerwünschte Wirkungen unter dem Antihypertensivum Debrisoquin und unter dem Antiarrhythmikum Spartein bei einzelnen Personen gefunden. Es zeigte sich, dass langsame Metabolisierer für Spartein ebenfalls langsame Metabolisierer für Debrisoquin waren. Dem zunächst unter dem Begriff Debrisoquin/SparteinPolymorphismus bezeichneten Phänomen konnte später das Cytochrom P 450 2D6 (CYP 2D6) zugeordnet werden $(2,17)$. Heute kennt man mehr als 50 verschiedene Pharmaka, die über das Cytochrom CYP 2D6 metabolisiert werden. Etwa 8\% der kaukasischen (westeuropäische und weiße amerikanische) Be-
Tab. 2 Nomenklatur des Cytochrom-P450-Systems.

Cytochrom P450-System umfasst eine Gruppe von Enzymen.

Die erste Zahl steht für die Genfamilie.

Der Buchstabe definiert die Subfamilie.

Die Zahl nach dem Buchstaben steht für das jeweilige Isoenzym.

völkerung sind sog. „poor metaboliser“, d.h. defizient für dieses Enzym, während sich bei etwa 1,5-5\% der kaukasischen Bevölkerung eine sehr hohe Aktivität des CYP 2D6 (ultraschnelle Metabolisierer) findet $(16,21)$.

\section{Das Cytochrom-P450-System und seine Bedeutung bei Arzneimittelinteraktionen}

Das Cytochrom-P450-System (Tab.2) umfasst eine Gruppe mischfunktioneller Oxygenasen, die ein Sauerstoffatom aus molekularem Sauerstoff auf ihr Substrat übertragen (Substrat wird oxidiert, Sauerstoff wird reduziert). Sie kommen hauptsächlich in der Leber vor, jedoch sind auch extrahepatische Manifestationen bekannt, von denen insbesondere das intestinale Vorkommen von Interesse ist, da hier Arzneistoffe bereits während der Passage der Darmschleimhaut einem Metabolismus unterliegen (13).

Den Genfamilien CYP 1 bis CYP 4 kommt beim Metabolismus von Fremdstoffen die größte Bedeutung zu, derzeit sind in diesen Genfamilien mehr als 20 Isoenzyme bekannt, von denen eine Vielzahl genetischer Polymorphismen bekannt ist. Nahezu $50 \%$ aller Arzneistoffe sowie endogene Substanzen wie Steroide und Sexualhormone werden über das CYP 3A4 metabolisiert. Eine vollständige Defizienz dieses Enzyms ist nicht bekannt, jedoch besteht eine große interindividuelle Variabilität in der Enzymaktivität, die z.T. auch in dessen spezifischer Induzierbarkeit durch Arznei- und Genussmittel begründet ist $(13,22)$. Eine vollständige Defizienz des CYP 2C19 findet sich bei etwa 3\% der europäischen Bevölkerung aber bei etwa $20 \%$ der asiatischen Bevölkerung $(6,8,22)$. Etwa 5-10\% der europäischen Bevölkerung weisen eine CYP 2D6-Defizienz auf, hingegen nur $1 \%$ der orientalischen Bevölkerung $(1,8,16,22)$.

Viele der klinisch eingesetzten Pharmaka werden nicht nur durch ein Isoenzym des Cytochromsystems metabolisiert, so kommt es zu einer Überlappung der Substratspezifität mit mehreren Isoenzymen, beispielsweise wird Phenytoin über CYP 2C19 und CYP 2C9 metabolisiert. Von einem einheitlichen Abbauweg einer Substanzklasse darf nicht zwingend ausgegangen werden ausgegangen werden, so wird Propranolol über CYP 2C19 metabolisiert (6), während die meisten Beta-Blocker über das CYP 2D6 abgebaut werden (16).

Auch ist die klinische Relevanz der polymorph exprimierten Cytochrome bei vielen Substanzen umstritten, vor allem bei Substanzen, die nicht monoenzymatisch verstoffwechselt werden (15). Ferner spielen das Ausmaß der Affinität und eine eventuelle Inhibition eine Rolle (13). Vor allem für die polymorph exprimierten Enzyme CYP 2C9, CYP 2C19 und CYP 2D6 liegen Untersuchungen zur klinischen Relevanz vor $(3,15,20)$ (Tab.3). 
Tab.3 Substratspezifität von Cytochrom 450-Enzymen - einige Beispiele (mod. n. )3,15,19,20)).

\begin{tabular}{|c|c|c|c|c|c|}
\hline CYP $1 A 2$ & CYP 2 C8 & CYP 2C9 & CYP $2 C 19$ & CYP 2D6 & CYP $3 A 4$ \\
\hline Coffein & Taxol & $\begin{array}{l}\text { NSAR (z.B. Ibuprofen, } \\
\text { Diclofenac, Naproxen) }\end{array}$ & Diazepam & Debrisoquin/Spartein & Ciclosporin \\
\hline Phenacetin & & Omeprazol & Spartein & $\begin{array}{l}\text { Antiarrhythmika (Propafenon, } \\
\text { Flecainid, Encainid, Ajmalin) }\end{array}$ & Hydrocortisone \\
\hline Paracetamol & Celecoxib & Pantoprazol & & Amphetamine & Estradiol \\
\hline Clozapine & Phenytoin & Hexobarbital & & $\begin{array}{l}\text { Betablocker (Metoprolol, } \\
\text { Carvedilol, Timolol) }\end{array}$ & Makrolide \\
\hline Tamoxifen & Warfarin & Phenobarbital & & Metoprolol & Tia-, Midazolam \\
\hline Theophyllin & Losartan & Proguanil & Phenytoin & trizyklische Antidepressiva & $\begin{array}{l}\text { - Ca-Antagonisten } \\
\text { (Verapamil, Diltiazem, } \\
\text { Nife-, Amlodipin...) }\end{array}$ \\
\hline Imipramin & & Imipramin & Moclobemid & $\begin{array}{l}\text { Neuroleptika (Haloperidol, } \\
\text { Risperidon, Perphenazin) }\end{array}$ & $\begin{array}{l}\text { die meisten CSE-Inhibitoren } \\
\text { (Lova-, Ceriva-, Atorvastatin) }\end{array}$ \\
\hline Ondansetron & & Mephenytoin & Propranolol & Ondansetron & Methadon \\
\hline Amitryptillin & & Irbesartan & Cyclophosphamid & (Dihydro-)Codein & Cisaprid \\
\hline Clomipramin & & Tolbutamid & Citalopram & Phenacetin & Lidocain \\
\hline \multirow[t]{8}{*}{ Estradiol } & & Glibenclamid & Nelfinavir & Lidocain & Quinidin \\
\hline & & Glipizide & Progesteron & Fluoxetin (auch CYP 3A4) & HIV-Protease-Inhibitoren \\
\hline & & Rosiglitazon & Indomethacin & Tamoxifen & Chlorpheniramin \\
\hline & & Amitryptillin & R-Warfarin & Clorpromazine & Astemizol \\
\hline & & Fluvastatin & & & Azol-Antimykotika \\
\hline & & Sulfamethoxazole & & & $\begin{array}{l}\text { Antimalariamittel } \\
\text { (Mefloquin, Halofantrin) }\end{array}$ \\
\hline & & S-Warfarin & & & Koffein - Kokain \\
\hline & & Phenprocoumon (?) & & & Vincristine \\
\hline
\end{tabular}

Einige Beispiele mit klinischer Bedeutung:

- Die Gabe von den Antiarrhythmika (Propafenon, Flecainid, Ajmalin, Prajmalin) kann bei Personen mit einer verminderten Aktivität des CYP 2D6 bereits in üblicher Dosierung zu erheblichen Nebenwirkungen und Symptomen der Intoxikation führen (16).

- Über das Cytochrom-System erfolgt bei einigen Arzneimitteln (sog. Prodrugs) die Aktivierung: so wird z.B. Codein als Prodrug verabreicht, für dessen Bioaktivierung zu Morphin (Demethylierung) das CYP 2D6 benötigt wird. Bei CYP 2D6defizienten Personen ist der analgetische Effekt des Codeins daher geringer ausgeprägt (2).

- Pharmakogenetisch bedingtes Therapieversagen kann bei extrem schnellem Abbau einer Substanz durch Hypermetabolismus auftreten, so zum Beispiel scheinbar therapierefraktäre Ulzera ventriculi et duodeni unter Therapie mit Omeprazol, wenn ein Polymorphismus des CYP 2C19 vorliegt (4), so dass die üblichen Dosen nicht mehr ausreichen (21).

\section{Methoden zur Untersuchung der genetischen Polymorphismen}

\section{Phänotypisierung}

Bei der In-vivo-Phänotypisierung wird eine Testsubstanz oder ein Cocktail aus verschiedenen Substanzen verabreicht (z.B. Dextrometorphan bei CYP 2D6-Metabolismus) und anschließend die Elimination oder die Bildung eines Metaboliten in Patientenproben (Blut, Speichel, Urin, Ausatemluft etc.) gemessen
(z.B. Messung des Dextrometorphan-Metaboliten Dextrorphan im Urin). Der Nachteil dieses Verfahren ist die Störanfälligkeit durch Noxen oder Co-Medikation. Wurde die medikamentöse Therapie bereits begonnen, ist die in-vivo-Phänotypisierung meist nicht mehr möglich (2).

Ex-vivo lässt sich beispielsweise die Aktivität der ThiopurinMethyltransferase, bei der eine Defizienz in der heterozygoten Form bei etwa 10\% der Bevölkerung vorliegt, im Plasma oder die der Alkohol-Dehydrogenase bestimmen (10). Dieses Verfahren wird allerdings nur in wenigen Zentren in Deutschland durchgeführt.

\section{Genotypisierung}

Mittels Genotypisierung, in der Regel aus Leukozyten, konnten verschiedene Allele bei demselben Phänotyp eines Enzymmangels identifiziert werden. Beispielsweise existieren beim CYP 2D6 neben dem so genannten Wildtyp noch > 70 weitere Allele am Genlocus, von denen einige ein vollständiges Fehlen des Enzyms oder eine verminderte Enzymaktivität und andere wiederum eine erhöhte Enzymaktivität bewirken $(16,21)$.

Diese Methode hat den Vorteil, dass Störfaktoren (z.B. Noxen oder Co-Medikation) das Testergebnis nicht beeinflussen können (2). Der Nachteil ist, dass sie nur eingeschränkt Rückschlüsse auf die in-vivo tatsächlich vorliegende enzymatische Aktivität gestattet.

Es wäre wünschenswert, wenn vor Beginn einer medikamentösen Therapie mit Medikamenten, die sowohl einem Stoffwech- 
selweg mit häufig vorkommenden Polymorphismen (z.B. CYP 2D6) unterliegen als auch eine geringe therapeutischen Breite haben (z.B. Azathioprin), eine Geno- und/oder Phänotypisierung erfolgen würde. Entsprechende Dosierungsvorschläge wurden von Kirchheiner et al. für Antidepressiva erarbeitet (8). In der Zukunft wird es möglicherweise routinemäßige Bestimmungen von erblichen Unterschieden im Fremdstoffmetabolismus vor Beginn einer Arzneimitteltherapie für ausgewählte Patienten oder vor Gabe bestimmter Pharmaka - z.B. Messung der Dihydropyrimidin-Dehydrogenase vor Therapie mit 5-Fluor-Uracil $(9,11)$ - geben (2). Abgesehen von der Tatsache, dass eine derartige Genotypisierung derzeit nur wenigen Zentren vorbehalten ist, fehlen hier noch Studien zur Kosten/Nutzen-Bewertung.

Bei Therapie mit Arzneimitteln, deren Metabolismus bekanntermaßen einem genetischen Polymorphismus unterliegt, sollte eine sorgfältige Dosistitration und gegebenenfalls therapeutisches Drug monitoring erfolgen, um den interindividuellen Unterschieden im Arzneimittelmetabolismus gerecht $\mathrm{zu}$ werden (15).

\section{kurzgefasst: Aufgrund der erheblichen interindividuellen Unterschiede im Arzneimittelmetabolismus bei Substra- ten von Enzymen, die einem genetischen Polymorphis- mus unterliegen, sind Dosistitration und eventuell the- rapeutisches Drug Monitoring erforderlich.}

\section{Literatur}

1 Brockmoeller J, Roots I. Assessment of liver metabolic function. Clinical implications. Clin Pharmacokinet 1994; 27: 216-248

2 Ensom MHH, Chang TKH, Patel P. Pharmacogenetics. The therapeutic drug monitoring of the future? Clin Pharmacokinet 2001; 40: 783802

3 Flockhart DA. Cytochrome P450 drug interaction table. http:// www.medicine.iupui.edu/flockhart. Last update, 2002

${ }^{4}$ Furuta T, Takashima M, Shirai N, Takashima M et al. Effect of genotypic differences in CYP 2 C19 on cure rates for helicobacter pylori infection by tripple therapy with a proton pump inhibitor, amoxicillin and clarithromycin. Clin Pharmacol Ther 2001; 69: 158-168

${ }^{5}$ Gsur A, Haidinger G, Hollaus P et al. Genetic polymorphism of CYPA1 and GSTM1 and lung cancer risk. Anticancer Res 2001; 21: 22372242

${ }^{6}$ Goldstein JA. Clinical relevance of genetic polymorphism in the human CYP2C subfamiliy. Br J Clin Pharmacol 2001; 52: 349-355

7 Han XM, Zhou HH. Polymorphism of CYP 450 and cancer susceptibility. Acta Pharmacol Sin 2000; 21: 672-679

8 Kirchheiner J et al. CYP2D6 and CYP2C19 genotype-based dose recommendations for antidepressants: a first step towards subpopulation specific dosages. Acta Psychiatr Scand 2001; 104: 173-192

${ }^{9}$ Kivisto KT, Kroemer HK, Eichelbaum M. The role of human cytochrome P450 enzymes in the metabolism of anticancer agents: implication for drug interactions. Br J Clin Pharmacol 1995; 40: 523-530

${ }^{10}$ Kröplin T, Weyer N, Gutsche S, Iven H. Thiopurine S-methyltransferase activity in human erythrocytes: a new HPLC-method using 6-thioguanine as substrate. Eur J Clin Pharmacol 1998; 54: 265-271

11 van Kuilenburg AB, Muller EW, Haasjes J et al. Clinical implications of dihydropyrimidine dehydrogenase deficiency in patients with severe 5-fluorouracil-associated toxicity. Clin Cancer Res 2000; 6: 47054712

12 Martinez C, Garcia-Martin E, Ladero JM et al. Association of CYP2C9 genotypes leading to high enzyme activity and colorectal cancer risk. Carcinogenesis 2001; 22: 1323-1326

13 Martin-Facklam M, Drewe J, Haefeli WE. Arzneimittel-Interaktionen am Cytochrom-P-450-System. Dtsch Med Wochenschr 2000; 3: 6367

${ }^{14}$ Neber DW, Roe AL. Ethic and genetic differences in metabolism genes and risk of toxicity and cancer. Sci Total Environ 2001; 274: 93-102

15 Phillips KA, Veenstra DL, Oren E, Lee JK, Sadee W. Potential role of pharmacogenomics in reducing adverse drug reactions. JAMA 2001; 286: 2270-2279

16 Pirmohamed M, Park BK. Genetic susceptibility to adverse drug reactions. Trends Pharmacol Sci 2001; 22: 298-305

17 Pirmohamed M. Pharmacogenetics and pharmacogenomics. Br J Clin Pharmacol 2001; 52: 345-347

${ }^{18}$ Rieder MJ, Shear NH, Kanee A, Tang BK, Spielberg SP. Prominence of a slow acetylator phenotype among patients with sulfonamide hypersensitivity reactions. Clin Pharmacol Ther 1991; 49: 13-17

19 Roots I, Brockmüller J. Pharmakogenetik. 4. Auflage, Steinkopf-Verlag Darmstatt, In: Rietbrock N, Staib AH, Loew D (Hrsg.) Klinische Pharmakologie - Arzneitherapie. 2001: 64-79

20 Schwab M, Marx C, Zanger UM, Eichelbaum M. Pharmakogenetik der Zytochrom-P-450-Enzyme. Dtsch Ärztebl 2002; 8: B400-B406

${ }^{21}$ Steijns LSW, van der Weide J. Ultrarapid drug metabolism: PCR based detection of CYP2D6 gene duplication. Clin Chem 1998; 44: 914-917

22 Xie HG, Kim RB, Wood AJJ, Stein M. Molecular basis of ethnic differences in drug disposition and response. Annu Rev Pharmacol Toxicol 2001; 41: 815-850 